\title{
Estudio palinológico en especies de Asplenium (Aspleniaceae) de Argentina
}

\author{
MARÍA ALEJANDRA GANEM${ }^{1}$, MARÍA LUJÁN LUNA²,3 y GABRIELA ELENA GIUDICE²
}

\begin{abstract}
Summary: Palynological study in Asplenium species (Aspleniaceae) from Argentina. The genus Asplenium L. is represented in Argentina by about 38 species, being its habit terrestrial, saxicolous or ephiphytic. In this contribution we complete the palynological study of the genus in this country. The material was processed according to standard techniques for observation under light and scanning electron microscopes. The spores are monolete, the wall formed by the homogeneous exospore, with smooth surface, and the camerate and folded perispore. Two types of folds, winged and ridged, are registered. A variability in the density of the perforations in the outer layer of the perispore, and the margin and sculptural elements of the folds and in the areas between them, are also observed. Spores of analyzed taxa are described and illustrated with pictures using scanning electron microscope.
\end{abstract}

Key words: Asplenium, spores, morphology, Argentina.

Resumen: El género Asplenium L. se halla representado en Argentina por cerca de 38 especies, siendo su hábito terrestre, saxícola o epífito. En la presente contribución se completa el estudio palinológico del género en este país. El material fue procesado de acuerdo a técnicas clásicas para su observación con microscopios óptico y electrónico de barrido. Las esporas son monoletes, la pared está conformada por el exosporio homogéneo de superficie lisa, y el perisporio camerado y plegado. Se distinguen dos tipos de pliegues: alados y lomados y se observa variabilidad en cuanto a la densidad de las perforaciones del estrato externo del perisporio, a los tipos de margen y los elementos esculturales de los pliegues y a las áreas entre pliegues. Se describen las esporas de los taxa estudiados y se ilustran con fotografías al microscopio electrónico de barrido.

Palabras clave: Asplenium, esporas, morfología, Argentina.

\section{INTRODUCCIÓN}

El género Asplenium se halla representado en Argentina por cerca de 38 especies, distribuidas principalmente en el Noroeste, Noreste y Centro, y solo tres en el área Patagónica. Son plantas de hábito terrestre, epifito o saxicola.

Para la Argentina, las esporas de las especies que crecen en el NO y Centro de Argentina fueron

${ }^{1}$ Cátedra de Botánica General, Facultad de Ciencias Agrarias, Universidad Nacional de Jujuy, Argentina; aleganem@hotmail.com

${ }^{2}$ Cátedra de Morfología Vegetal, Facultad de Ciencias Naturales y Museo, Universidad Nacional de La Plata, Argentina; gegiudice@hotmail.com

${ }^{3}$ Comisión de Investigaciones Científicas de la Provincia de Buenos Aires, Camino Gral. Belgrano y 526, CP 1900 , La Plata, Argentina; lujanluna@fcnym.unlp.edu.ar estudiadas por Giudice et al. (2002) y Morbelli \& Giudice (2005). En el primer trabajo, donde se analiza la morfología y escultura de las esporas con microscopía óptica y electrónica de barrido, se reconoce para todos los taxa un perisporio plegado, con variabilidad en la densidad de las perforaciones y el tipo y margen de los pliegues. En el segundo trabajo se estudia con microscopía electrónica de transmisión la ultrestructura de la esporodermis, la cual está conformada por exosporio liso con dos capas y perisporio también con dos capas, con una organización estructural cavada o camerada.

En lo que respecta a las 3 especies que habitan en la Patagonia argentina, A. monanthes crece también en centro y NOA, por lo que sus esporas ya fueron estudiadas en trabajos previos (Giudice et al., 2002).

Las esporas de $A$. scandicinum, taxón que crece en la provincia de Misiones, fueron analizadas por 
Ganem et al. (2001) en su contribución sobre el grupo Asplenium squamosum en América.

Asplenium laetum y A. triquetrum, especies citadas para la Argentina (Ponce, 1996), eran consideradas dentro de la sección Hymenasplenium (Murakami \& Moran, 1993). De acuerdo a los últimos estudios morfológicos y moleculares (Murakami, 1995; Christenhusz et al., 2011; Gabancho \& Prada, 2011) se reconoce a Hymenasplenium como género. Las especies de Hymenasplenium que crecen en Argentina y la morfología de sus esporas son tratadas por Giudice et al. (En prensa).

Tryon (1990) encuentra una relación entre el hábito de las plantas y la ornamentación de las esporas, observando que las epifitas tienen una ornamentación más compleja que las terrestres o saxicolas.

El objetivo de este estudio es analizar la morfología y escultura de la pared de las esporas en los taxa de Asplenium que crecen en Argentina y que no han sido abordados hasta el presente, completando de este modo la información palinológica para el género en el país. Se pretende contribuir a la sistemática del género y de la familia Aspleniaceae, e indagar sobre la posible relación entre los caracteres de las paredes de las esporas y el hábito de los helechos.

\section{Materiales y Métodos}

El estudio se realizó con material de herbario y fresco. Los especimenes de herbario corresponden a las siguientes Instituciones (siglas según Thiers, 2012): LP, MERL, SI y Herbario JUA.

Las observaciones se realizaron con microscopios óptico (MO) y electrónico de barrido (MEB). En ambos casos el material fue analizado sin tratamiento químico previo.

Para las observaciones al MO se utilizó un microscopio Nikon Labophot-2. Se calcularon las siguientes medidas promedio entre 20 esporas: diámetro ecuatorial mayor, diámetro ecuatorial menor, diámetro polar, longitud de lesura y altura de los pliegues.

Para el estudio al MEB las muestras fueron metalizadas con oro-paladio. Las observaciones se realizaron en un microscopio JEOL JSMT-100.

La terminología empleada en la Tabla 1 se refiere a la considerada por Giudice et al.(2002).
Material representativo estudiado

A. abscissum Willd. ARGENTINA. Prov. Misiones, Dpto. Cainguás, Arroyo Tateto, Márquez 46 (LP).

A. alatum Willd. BRASIL. Edo. Rio Grande do Sul, Rosenstock 28 (SI).

A. auriculatum Sw. ARGENTINA. Prov. Misiones, Dpto. Iguazú, Eskuche 1760 (SI).

A. balansae (Baker) Sylvestre. ARGENTINA. Prov. Misiones, Dpto Iguazú, Zuloaga et al 5630 (SI).

A. brasiliensis Sw. ARGENTINA. Prov. Misiones: Dpto San Pedro, Parque Provincial Moconá, Marquez 106 (LP).

A. claussenii Hieron. ARGENTINA. Prov. Misiones, Dpto. San Pedro, R. P. Caa Yari, Arroyo Paraiso, Marquez 292 (LP).

A. cuspidatum Lam. ARGENTINA. Prov. Tucumán, Dpto. Famaillá, Castillon s/no (SI 46037).

A. dareoides Desv. ARGENTINA. Prov. Chubut, Parque Nacional Lago Puelo, Vidoz 121 (LP).

A. gastonis Fée. ARGENTINA. Prov. Misiones, Dpto. San Pedro, R. P. Caa Yari, Marquez 241 (LP).

A. inaequilaterale Willd. ARGENTINA. Prov. Misiones, Dpto. Cainguás, Biganzoli et. al. 1377 (SI).

A. kunzeanum Klotzsch ex Rosenst. ARGENTINA. Prov. Misiones, Dpto. San Pedro, R. P. Caa Yari, Marquez 249 cA (LP).

A. mucronatum C. Presl. PARAGUAY. Dpto. San Pedro, Alboff s/no (LP 18971).

A. oligophyllum Kaulf. BRASIL. Edo. Rio Grande do Sul, Rosenstock 30 (SI).

A. papaverifolium (Kunze) Viane. ARGENTINA. Prov. Mendoza, Dpto. Tupungato, Ruiz Leal 4599 (MERL).

A. pulchellum Raddi. ARGENTINA. Prov. Misiones, Campo Cumprido, C. Spegazzini s/n (SI 18439).

A. radicans L. ARGENTINA. Prov. Misiones, Dpto. Iguazú, Alto Paraná, Riemel 120 (SI).

A. sellowianum (Hieron.) C. Presl ex Hieron. ARGENTINA. Prov. Buenos Aires, Pdo. Ensenada, Reserva Natural Punta Lara, Ramos Giacosa 13 (LP).

A. serratum L. ARGENTINA. Prov. Misiones, Dpto. Eldorado, Burkart 14731 (SI).

A. trilobum Cav. CHILE. X Región de Los Lagos, Llanquihue, Werdermann 77 (SI).

A. ullbrichtii Rosenst. ARGENTINA. Prov. Buenos Aires, Pdo. Ensenada, Reserva Natural Punta Lara, Ramos Giacosa 12 (LP). 
A. uniseriale Raddi. ARGENTINA. Prov. Misiones, Dpto. San Pedro, Parque Provincial Moconá, Marquez \& Yañez 51 (LP).

\section{Resultados}

En la Tabla 1 se presentan los elementos y las características de la ornamentación de las esporas por especie y el hábito de estas plantas, y en la Tabla 2 se expresan las medidas obtenidas de las esporas.

En todos los casos las esporas son monoletes, elípticas en vista polar y planas a cóncavo- convexas en vista ecuatorial, de 30-50 $\mu \mathrm{m}$ de diámetro ecuatorial y de 20-40 $\mu \mathrm{m}$ de diámetro polar (Figs. 1-6). La lesura es tenuimarginada y generalmente se distingue un pliegue supralesural (Fig. 1D; Fig. 2 E).

El exosporio en sección es una capa homogénea de 1.5- $3 \mu \mathrm{m}$ de espesor y superficie lisa (Fig. $1 \mathrm{~L}$ ).

El perisporio tiene un espesor de 1-10 $\mu \mathrm{m}$, variabilidad dada por la diferencia de altura de los pliegues. En la mayoría de los casos presenta una estructura camerada, con tres estratos en sección (Fig. 1C; Fig. 2 F; Fig. 4 H; Fig.6 C). El estrato interno es continuo y delgado; el estrato medio está formado por columnas que dejan espacios o cámaras entre ellas y el estrato externo es delgado, generalmente perforado y porta los elementos de la escultura. En algunas fracturas (Asplenium gastonis, Fig. 3 B) no se observa el estrato medio con columnas.

El perisporio muestra una ornamentación variada, encontrándose pliegues en forma de alas o de lomos. El margen de los pliegues puede ser liso, dentado o espinoso. En este último caso las espinas miden entre 5-10 $\mu \mathrm{m}$ (A. auriculatum, Fig. $1 \mathrm{G}, \mathrm{H})$.

Se observan perforaciones de diferente tamaño y patrón de densidad, y diversos elementos esculturales cubren la superficie, como rúgulas, espinulas y crestas, los cuales presentan distinta densidad y tamaño según las especies.

\section{Discusión y Conclusiones}

Teniendo en cuenta las características de la escultura, las esporas estudiadas pueden reunirse en dos grupos de acuerdo a los tipos de pliegues: aladas y lomadas. Se registra además una variación entre grupos respecto a la densidad de las perforaciones del estrato externo del perisporio, en las características de los márgenes de los pliegues y en los elementos esculturales de los pliegues y de las áreas entre pliegues. Lo hallado en el presente estudio en cuanto a los tipos básicos de ornamentación del perisporio en esporas de Asplenium sp. coincide con la información aportada por Giudice et al. (2002).

En cuanto a las especies australes: A monanthes, A. daereoides y A. trilobum, en todos los casos las esporas son aladas-equinuladas, y fenestradas entre pliegues, si bien este tipo de ornamentación no es exclusivo del grupo austral.

Las dimensiones mayores de las esporas de Asplenium radicans podrían estar relacionadas con la poliplodía, ya que de acuerdo a Tryon \& Tryon (1982) y Mickel \& Smith (2004) se registran en esta especie tetraploides y octoploides $(2 \mathrm{n}=144,216$, 288).

La ornamentación de las esporas de las especies estudiadas aporta información para la sistemática, permitiendo la identificación a nivel específico en algunos taxa de Asplenium (A. auriculatum y $A$. serra) o de grupos de especies dentro de las Aspleniaceae. Así de acuerdo a estudios realizados recientemente (Giudice et al. en prensa), en el género Hymenasplenium se encuentran dos tipos de ornamentación espinosa y plegado/ alada. La ornamentación espinosa es característica de un grupo de especies de Hymenasplenium, denominado por Murakami \& Moran (1993) como "grupo repandulum" mientras que la ornamentación plegado-alada se encuentra tanto en el género Hymenasplenium como Asplenium.

Con respecto a la estructura del perisporio, se puede inferir por las fracturas observadas, que en la mayoría de los casos la estructura es camerada, en tanto que en el caso de Asplenium gastonis, se observa una estructura cavada (Morbelli et al., 2005). Así, se podría relacionar la ornamentación con pliegues alados con una estructura del perisporio camerada y en aquellas con pliegues lomados, una estructura cavada. Se pretende continuar el estudio con microscopía electrónica de transmisión para poder profundizar el análisis ultraestructural de estas paredes esporales.

Con respecto a la relación entre las características de la pared de las esporas y el hábito de las plantas y los ambientes donde crecen, en este estudio se observa que las especies epifitas 
Bol. Soc. Argent. Bot. 48 (3-4) 2013
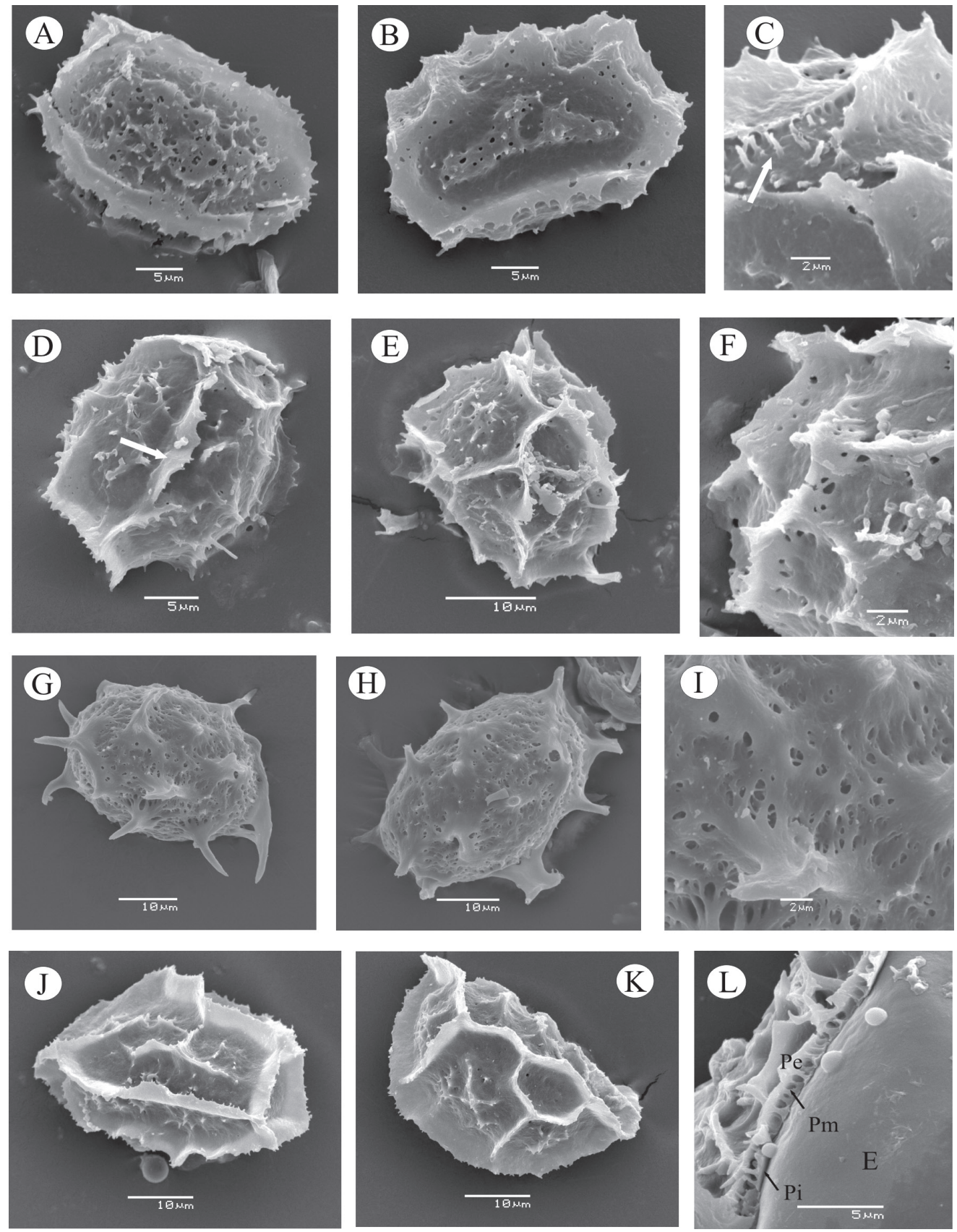

Fig. 1. Esporas vistas al MEB. A-C: Asplenium abscisum (Márquez et al. 46, LP). A: Vista distal. B: Vista ecuatorial. C: Detalle de pliegues con perforaciones y dientes en el margen y fractura del perisporio donde se observan las columnas del estrato medio (flecha). D- F: $A$. alatum (Rosenstock s/nº, SI 155628). D: Vista proximal, pliegue supralesural (flecha). E: Vista distal. Áreas entre pliegues con perforaciones y espínulas. F: Detalle de superficie con perforaciones y pliegues con dientes en el margen. G-I: A. auriculatum (Eskuche 1760, SI). G: Vista ecuatorial, pliegues con espinas. H: Vista distal. I: Superficie entre pliegues fenestrada, con abundantes perforaciones. J- L: A. balansae (Marquez et al. 70, SI). J: Vista proximal. K: Vista ecuatorial. L: Exosporio $(\mathrm{E})$ y fractura del perisporio: estrato interno $(\mathrm{Pi})$, estrato medio camerado $(\mathrm{Pm})$, estrato externo $(\mathrm{Pe})$. 



Fig. 2. Esporas vistas al MEB. A-C: A. brasiliensis (Marquez 106, LP). A: Vista distal. B: Vista ecuatorial. C: Detalle de pliegues con margen dentado y área entre pliegues con espinas y pliegues menores. D-F: Asplenium clausenii (Marquez 292, LP). D: Vista ecuatorial. E: Vista proximal, lesura con un pliegue supralesural (flecha). F: Detalle de fractura donde se observan las columnas (flecha) del estrato medio del perisporio y estrato externo con perforaciones. G- I: A. cuspidatum (Castillón $\mathrm{s} / \mathrm{n}^{\circ}, \mathrm{SI}$ ). G: Vista proximal. H: Vista distal. I: Vista ecuatorial. J-L: A. dareoides (Vidoz 121, LP). J: Vista ecuatorial. K: Vista proximal. L: Detalle de pliegues con margen dentado y área entre pliegues fenestrada. 
Bol. Soc. Argent. Bot. 48 (3-4) 2013
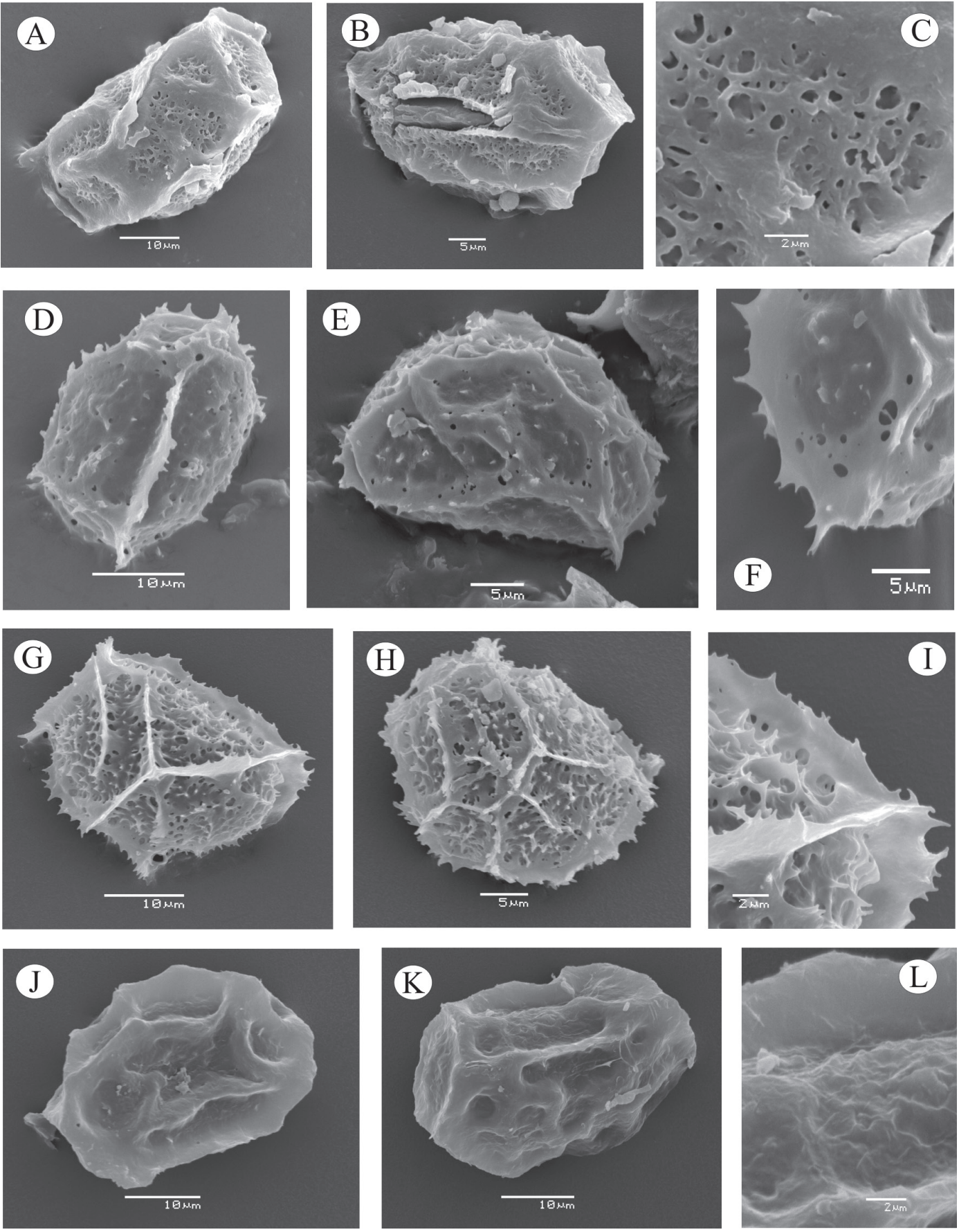

Fig. 3. Esporas vistas al MEB. A-C: A. gastonis (Marquez 241, LP). A: Vista distal con pliegues lomados. B: Vista proximal, fractura a nivel de la lesura. C: Detalle de área fenestrada entre pliegues. D-F: Asplenium inaequilaterale (Ramos Giacosa 125, LP). D: Vista proximal. E: Vista ecuatorial. F: Detalle de pliegues con dientes en el margen y grandes perforaciones. G- I: A. kunzeanum (Marquez 249, LP). G: Vista ecuatorial, pliegues parcialmente fusionados. $\mathbf{H}$ : Vista distal. I: Detalle de pliegues con margen dentado y áreas entre pliegues con dientes y perforaciones. J-L: A. mucronatum (Alboff $\mathrm{s} / \mathrm{n}^{\circ}$, LP 18971). J: Vista distal. K: Vista proximal. L: Pliegues de margen entero y superficie con rúgulas. 

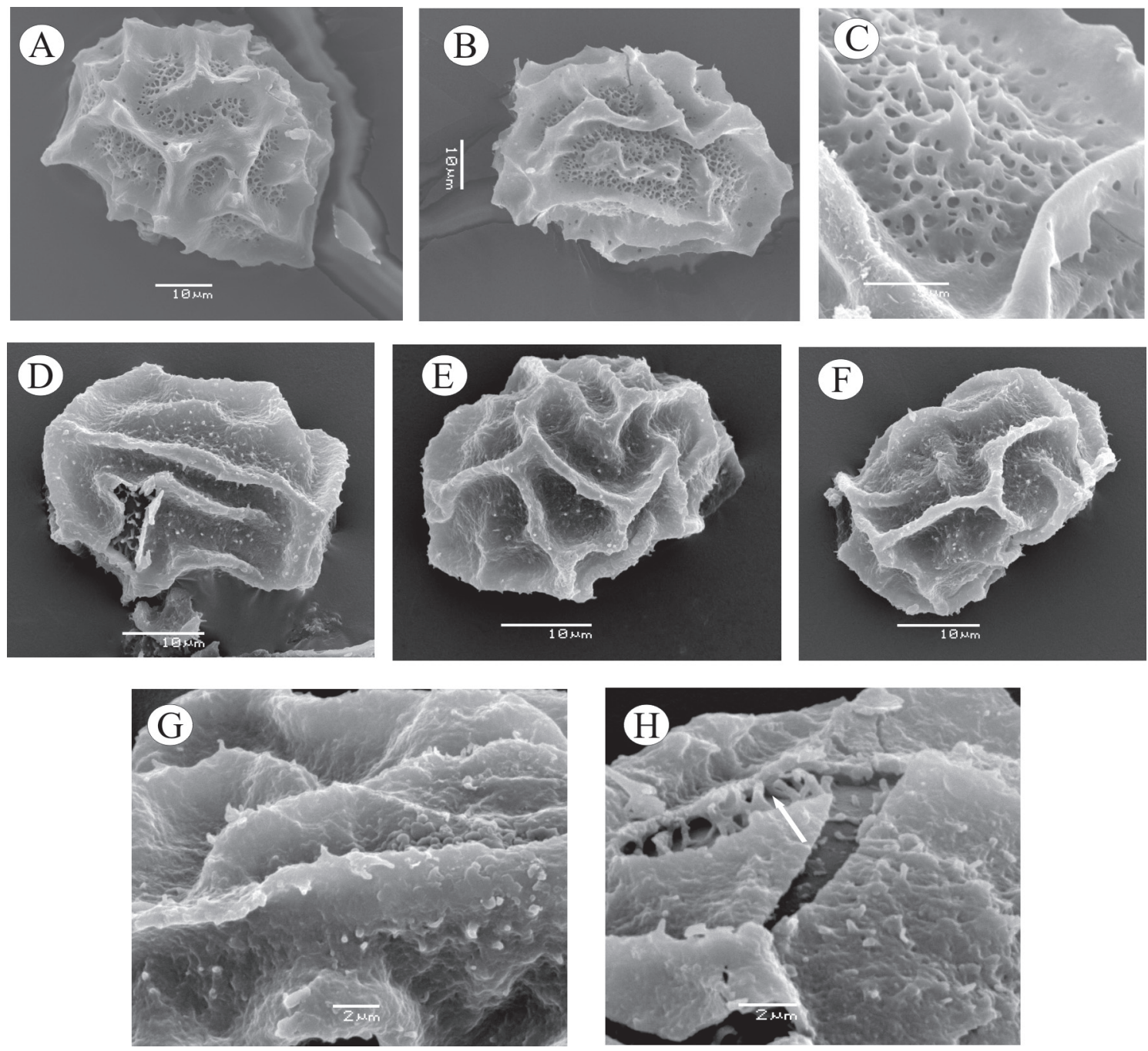

Fig. 4. Esporas vistas al MEB. A- C: A. oligophyllum (Rosenstock 30, SI). A: Vista distal. B: Vista ecuatorial. C: Área entre pliegues fenestrada. D-H: Asplenium papaverifolium (Ruiz Leal 4599, MERL). D: Vista proximal. E: Vista distal F: Vista ecuatorial. G: Detalle de superficie de pliegues con equínulas. H: Fractura del perisporio donde se observan las columnas del estrato medio (flecha).

tienen esporas con perisporio fenestrado o muy perforado, lo cual concuerda con lo mencionado por Tryon (1990) respecto a la mayor complejidad en la ornamentación del perisporio en epifitas de Asplenium. Estas características permitirían plantear que las esporas de las epífitas son más livianas y con paredes más permeables al agua, factor importante para el proceso germinativo. A pesar de ello se considera que son necesarios otros estudios que contribuyan a interpretar el rol adaptativo y ecológico de la ornamentación de las esporas de los helechos.
Con este estudio se completa la caracterización de las esporas de las especies de Asplenium que habitan en Argentina, aportando valiosa información para estudios sistemáticos, filogenéticos y biogeográficos.

\section{Agradecimientos}

Este trabajo se realizó en el marco de los proyectos de CONICET (PIP 878), UNLP (N610) y ANPCyT (PICT 661). 
Bol. Soc. Argent. Bot. 48 (3-4) 2013
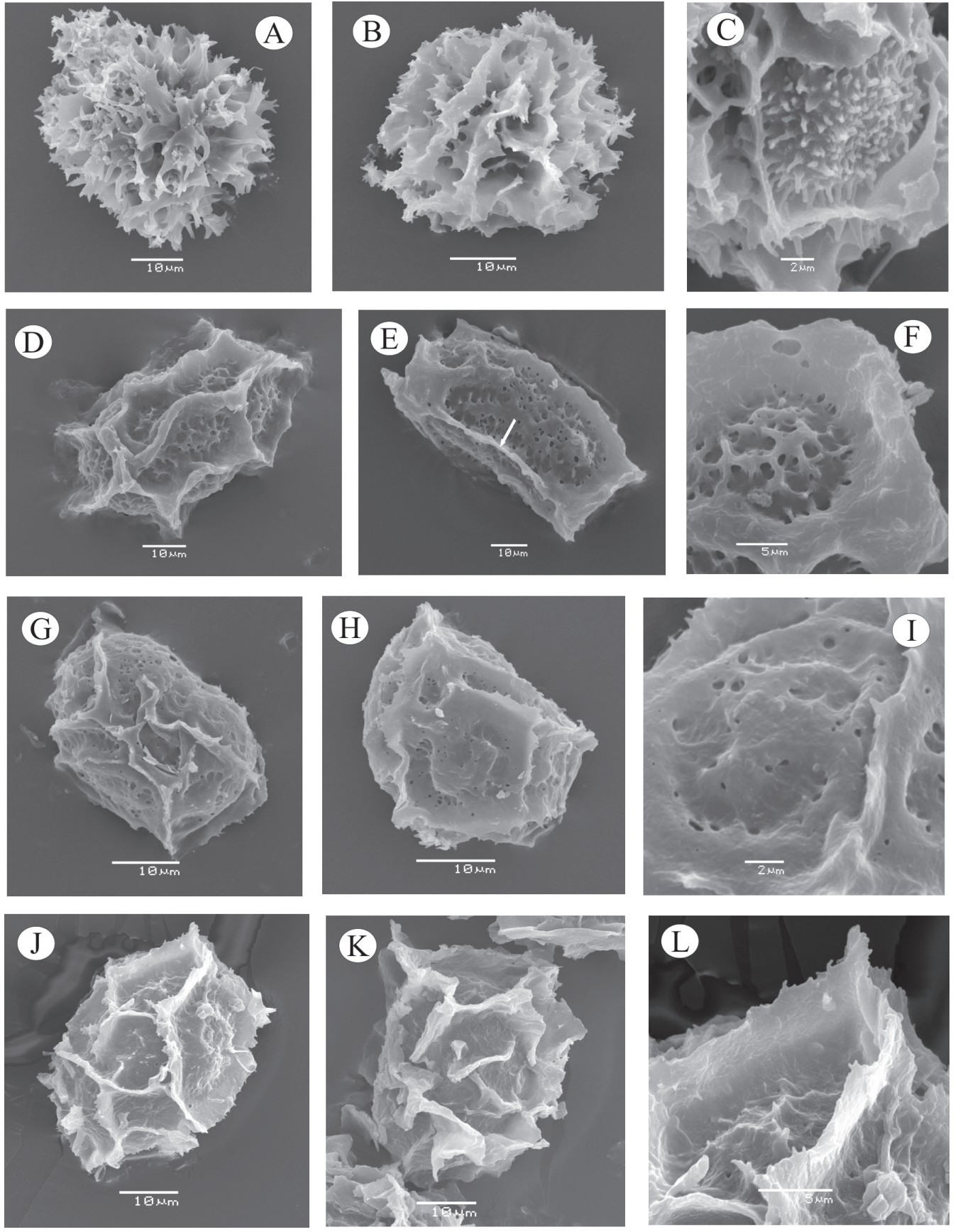

Fig. 5. Esporas vistas al MEB. A-C: Asplenium pulchellum (SI 18439). A: Vista distal con abundantes pliegues alados. B: Vista proximal con abundantes pliegues de margen equinado y perforados. C: Fractura del perisporio donde se observan las columnas del estrato medio. D- F: A. radicans (Riemels 120, SI). D: Vista distal. E: Lesura con un pliegue supralesural (flecha). F: Detalle de área entre pliegues fenestrada. G-I: A. sellowianum (Ramos Giacosa 13, LP). G: Vista distal. H: Vista ecuatorial. I: Detalle donde se observan perforaciones sobre los pliegues y en las áreas entre ellos. J-L: A. serratum (Burkart 14731, SI). J: Vista distal. K: Vista ecuatorial. L: Detalle de superficie con perforaciones ocasionales y pliegues con pequeños dientes en el margen. 

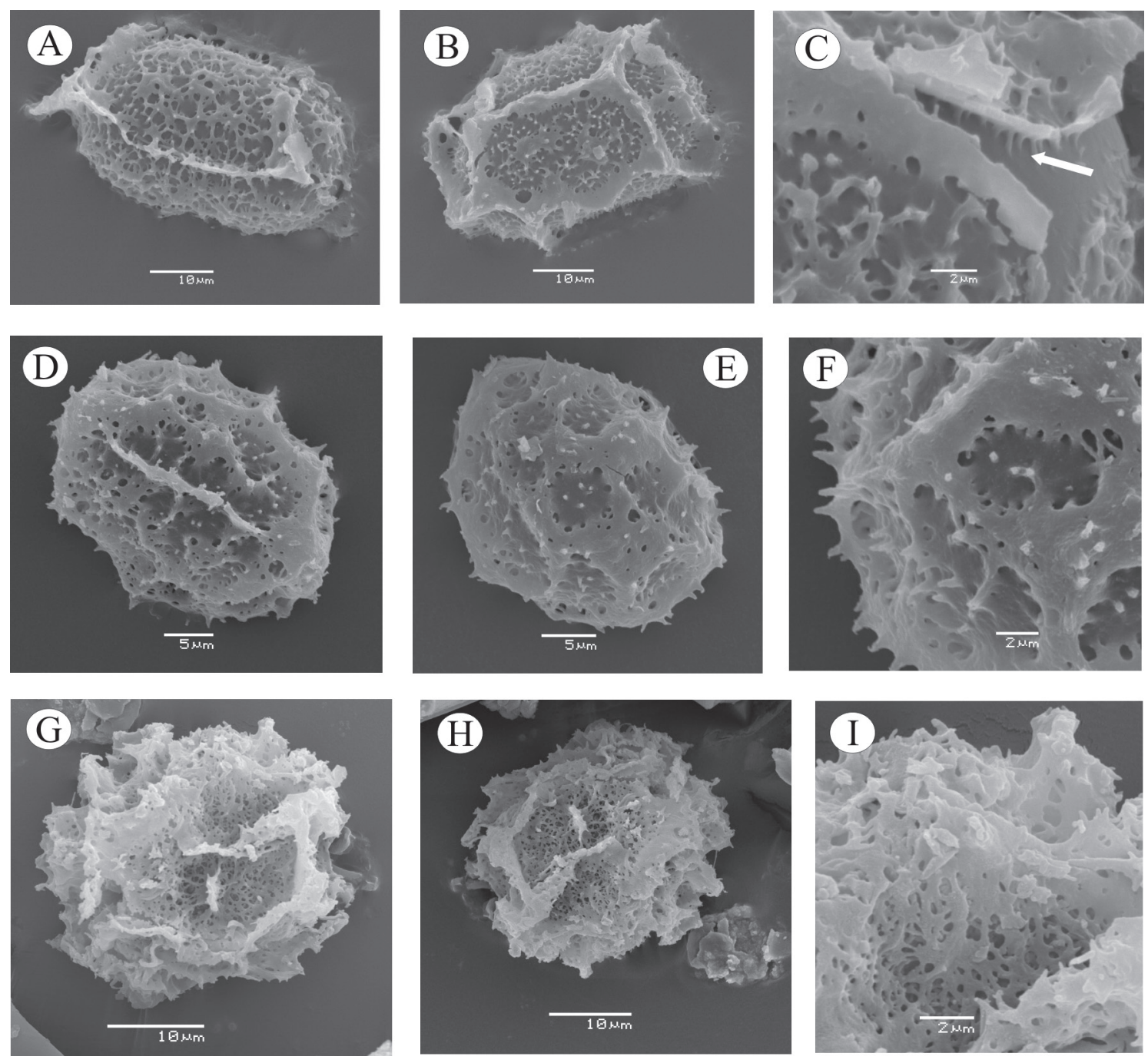

Fig. 6. Esporas vistas al MEB. A- C: Asplenium trilobum (Werdermann 77, SI). A: Vista proximal. B: Vista distal. C: Detalle de superficie y fractura del perisporio, donde se observa el estrato medio camerado (flecha). D-F: A. ullbritchii (Ramos Giacosa 12, LP). D: Vista proximal. E: Vista distal. F: Detalle de superficie, margen de pliegues con espinas. G-I: A. uniseriale (Márquez \& Yañez 51, LP). G: Vista distal. H: Vista proximal. I: Detalle de pliegues y superficie fenestrada.

\section{Biblografía}

CHRISTENHUSZ, M. J. M., X. ZHANG \& H. SCHNEIDER. 2011. A linear sequence of extant families and genera of lycophytes and ferns. Phytotaxa 19: 7-54.

GANEM, M.A., MORBELLI, M.A., GIUDICE, G.E., PIÑEIRO, M.R. \& I.G. MICHELENA. 2001. Las esporas del grupo Asplenium squamosum en América (Aspleniaceae, Pteridophyta). Polen 11: 51-58.

GIUDICE, G. E., MORBELLI, M.A. \& M.R. PIÑEIRO.
2002. Palynological study in Aspleniaceae from North-west Argentina. Bol. Soc. Argent Bot. 37: 217229.

GIUDICE, G.E., LUNA, MARÍA L., GANEM, M. ALEJANDRA. El género Hymenasplenium (Aspleniaceae) en Argentina. En prensa Darwiniana, Nueva Serie.

GABANCHO, L. R. \& C. PRADA. 2011. The genus Hymenasplenium (Aspleniaceae) in Cuba, including new combinations for the neotropical species. Amer. Fern J. 101: 265-281. 
Bol. Soc. Argent. Bot. 48 (3-4) 2013

MICKEL, J. T \& A.R. SMITH, 2004. The pteridophytes of Mexico. Mem. New York Bot. Gard. 88: 1-1054.

MORBELLI, M. A \& G. E. GIUDICE.2005. Spore wall ultrastructure in Aspleniaceae (Pteridophyta) from North-West Argentina. Rev. Palaeobot.Palynol. 135: 131- 143.

MURAKAMI, N. \& R. C. MORAN. 1993. Monograph of the Neotropical species of Asplenium sect. Hymenasplenium (Aspleniaceae). Ann. Mo.Bot. Gar. 80:1-38.

MURAKAMI, N. 1995. Systematics and evolutionary Biology of the Fern Genus Hymenasplenium (Aspleniaceae). J.Plant Res. 108: 257-268.

PONCE, M.1996. Pteridophyta. in Zuloaga, F. \& O. Morrone (eds.), Catálogo de las Plantas vasculares de la Rep. Argentina I. Monogr. Syst. Bot. Missouri Bot. Gard. 60: 1: 79.
THIERS, B.M. (ed.) 2012. Index Herbariorum. New York Botanical Garden, New York. (http://sciweb.nybg. org/science2/IndexHerbariorum.asp).

TRYON, R.M. \& A. F. TRYON. 1982. Ferns and Allied Plants. With Special Reference to Tropical America. Springer-Verlag, New York.

TRYON, A., 1990. Fern spores: evolutionary levels and ecological differentiation. Plant Syst. Evol. (Suppl. 5): $71-79$.

Recibido el 16 de noviembre de 2012, aceptado el 24 de junio de 2013. 
Tabla 1. Características del perisporio y hábito de las especies de Asplenium

\begin{tabular}{|c|c|c|c|c|c|c|c|}
\hline \multirow{3}{*}{ Especies } & \multicolumn{6}{|c|}{ PERISPORIO } & \multirow{3}{*}{ Hábito } \\
\hline & \multicolumn{4}{|c|}{ Pliegues } & \multirow{2}{*}{ Perforaciones } & \multirow{2}{*}{ Escultura } & \\
\hline & Tipo & Margen & Densidad & Fusión & & & \\
\hline $\begin{array}{l}\text { A abscissum } \\
\text { (Fig. 1 A-C) }\end{array}$ & alado & dentado & escasos & total & $\begin{array}{l}\text { abundantes } \\
\text { entre y sobre } \\
\text { pliegues }\end{array}$ & rúgulas & terrestre \\
\hline $\begin{array}{l}\text { A. alatum } \\
\text { (Fig. } 1 \mathrm{D}-\mathrm{F})\end{array}$ & alado & dentado & escasos & total & $\begin{array}{l}\text { abundantes en } \\
\text { base de pliegue }\end{array}$ & rúgulas espinas & epifita \\
\hline $\begin{array}{l}\text { A. auriculatum } \\
\text { (Fig. } 1 \mathrm{G}-\mathrm{I})\end{array}$ & alado & espinoso & escasos & parcial & $\begin{array}{l}\text { fenestrado } \\
\text { entre pliegues }\end{array}$ & & epifita \\
\hline $\begin{array}{l}\text { A.balansae } \\
\text { (Fig. } 1 \mathrm{~J}-\mathrm{L})\end{array}$ & alado & dentado & escasos & parcial & $\begin{array}{l}\text { escasas entre } \\
\text { pliegues }\end{array}$ & rúgulas/ espinas & terrestre \\
\hline $\begin{array}{l}\text { A. brasiliensis } \\
\text { (Fig. } 2 \text { A-C) }\end{array}$ & alado & dentado & escasos & total & ocasionales & $\begin{array}{l}\text { pliegues menores } \\
\text { y espinas }\end{array}$ & terrestre \\
\hline $\begin{array}{l}\text { A. claussenii } \\
\text { (Fig. } 2 \text { D-F) }\end{array}$ & alado & dentado & escasos & total & $\begin{array}{l}\text { abundantes e/ y } \\
\text { sobre pliegues }\end{array}$ & rúgulas y equinulas & terrestre \\
\hline $\begin{array}{l}\text { A. cuspidatum } \\
\text { (Fig. } 2 \text { G- I) }\end{array}$ & alado & entero & escasos & tota & $\begin{array}{l}\text { fenestrado } \\
\text { e/ pliegues }\end{array}$ & & epifita \\
\hline $\begin{array}{l}\text { A. dareoides } \\
\text { (Fig. } 2 \mathrm{~J}-\mathrm{L} \text { ) }\end{array}$ & alado & dentado & escasos & total & $\begin{array}{l}\text { fenestrado } \\
\text { e/ pliegues }\end{array}$ & espinas & $\begin{array}{l}\text { epifita, } \\
\text { saxicola, } \\
\text { terrestre }\end{array}$ \\
\hline $\begin{array}{l}\text { A. gastonis } \\
\text { (Fig. } 3 \text { A-C) }\end{array}$ & lomado & entero & escasos & total & $\begin{array}{l}\text { fenestrado } \\
\text { e/ lomos }\end{array}$ & & epifita \\
\hline $\begin{array}{l}\text { A. inaequilaterale } \\
\text { (Fig. } 3 \mathrm{D}-\mathrm{F} \text { ) }\end{array}$ & Iomado & entero & escasos & parcial & ocasionales & rúgulas & terrestre \\
\hline $\begin{array}{l}\text { A. kunzeanum } \\
\text { (Fig. } 3 \text { G-I) }\end{array}$ & alado & dentado & escasos & parcial & $\begin{array}{l}\text { fenestrado } \\
\text { e/ pliegues }\end{array}$ & equinulas y rúgulas & epifita \\
\hline $\begin{array}{l}\text { A. mucronatum } \\
\text { (Fig. } 3 \mathrm{~J}-\mathrm{L} \text { ) }\end{array}$ & alado & entero & abundantes & total & pocas & rugulas & epifita \\
\hline $\begin{array}{l}\text { A. oligophyllum } \\
\text { (Fig. } 4 \text { A-C) }\end{array}$ & alado & $\begin{array}{l}\text { ocasionales } \\
\text { dientes }\end{array}$ & abundantes & total & $\begin{array}{l}\text { fenestrado } \\
\text { e/ pliegues }\end{array}$ & & terrestre \\
\hline $\begin{array}{l}\text { A. papaverifolium } \\
\text { (Fig. } 4 \mathrm{D}-\mathrm{H} \text { ) }\end{array}$ & lomado & $\begin{array}{l}\text { escasos } \\
\text { dientes }\end{array}$ & abundantes & parcial & ocasionales & $\begin{array}{l}\text { rúgulas/ } \\
\text { equinulas }\end{array}$ & terrestre \\
\hline $\begin{array}{l}\text { A. pulchellum } \\
\text { (Fig. } 5 \text { A-C) }\end{array}$ & alado & dentado & $\begin{array}{l}\text { abundantes, } \\
\text { cubren la } \\
\text { superficie } \\
\text { totalmente }\end{array}$ & total & fenestrado & & terrestre \\
\hline $\begin{array}{l}\text { A. radicans } \\
\text { (Fig. } 5 \mathrm{D}-\mathrm{F} \text { ) }\end{array}$ & alado & entero & escasos & total & $\begin{array}{l}\text { fenestrada } \\
\text { e/ pliegues }\end{array}$ & & epifita \\
\hline $\begin{array}{l}\text { A. sellowianum } \\
\text { (Fig. } 5 \mathrm{G}-\mathrm{I} \text { ) }\end{array}$ & alado & dentado & escasos & parcial & $\begin{array}{l}\text { abundantes } \\
\text { e/ pliegues }\end{array}$ & rúgulas & $\begin{array}{l}\text { terrestre/ } \\
\text { ocasional } \\
\text { epifita }\end{array}$ \\
\hline $\begin{array}{l}\text { A. serratum } \\
\text { (Fig. } 5 \mathrm{~J}-\mathrm{L} \text { ) }\end{array}$ & alado & dentado & abundantes & parcial & ocasionales & $\begin{array}{l}\text { rúgulas equinulas y } \\
\text { plieges pequeños }\end{array}$ & epifita \\
\hline $\begin{array}{l}\text { A. trilobum } \\
\text { (Fig. } 6 \text { A-C) }\end{array}$ & alado & dentado & escasos & total & $\begin{array}{l}\text { fenestrada } \\
\text { e/ pliegues y } \\
\text { perforaciones } \\
\text { en pliegues }\end{array}$ & equinulas & epifita \\
\hline $\begin{array}{l}\text { A. ullbricthii } \\
\text { (Fig. } 6 \text { D-F) }\end{array}$ & alado & dentado & abundantes & total & $\begin{array}{l}\text { abundantes } \\
\text { sobre y e/ } \\
\text { pliegues }\end{array}$ & rúgulas y equinulas & terrestre \\
\hline $\begin{array}{l}\text { A. uniseriale } \\
\text { (Fig. } 6 \mathrm{G}-\mathrm{I} \text { ) }\end{array}$ & alado & dentado & abundantes & total & $\begin{array}{l}\text { fenestrada } \\
\text { e/ pliegues y } \\
\text { perforaciones } \\
\text { en pliegues }\end{array}$ & & $\begin{array}{l}\text { terrestre/ } \\
\text { epifita }\end{array}$ \\
\hline
\end{tabular}


Bol. Soc. Argent. Bot. 48 (3-4) 2013

Tabla 2. Medidas obtenidas en las esporas (valores en $\mu \mathrm{m}$ )

\begin{tabular}{|lccccc|}
\multicolumn{1}{c}{ Especie } & $\begin{array}{c}\text { Diámetro } \\
\text { Ecuatorial mayor }\end{array}$ & $\begin{array}{c}\text { Diámetro } \\
\text { Ecuatorial menor }\end{array}$ & $\begin{array}{c}\text { Diámetro } \\
\text { polar }\end{array}$ & $\begin{array}{c}\text { Longitud Lesura } \\
\text { A. abscisum }\end{array}$ & $\begin{array}{c}\text { Altura } \\
\text { pliegues }\end{array}$ \\
A. alatum & $38-50$ & $30-32$ & $26-28$ & $34-35$ & $5-6$ \\
A. auriculatum & $30-40$ & $20-22$ & $20-22$ & $16-23$ & $3-4$ \\
A. balansae & $35-40$ & $23-26$ & $24-25$ & $16-17$ & $2-3$ \\
A. brasiliensis & $40-42$ & $28-32$ & $25-27$ & $30-33$ & $4-5$ \\
A. claussenii & $38-40$ & $28-30$ & $25-27$ & $16-22$ & $4-6$ \\
A. cuspidatum & $29-35$ & $22-24$ & $20-22$ & $16-18$ & $2-4$ \\
A. dareoides & $40-50$ & $25-30$ & $28-30$ & $15-20$ & $2-3$ \\
A. gastonis & $38-69$ & $19-20$ & $20-22$ & $16-18$ & $3-7$ \\
A. inaequilaterale & $40-45$ & $25-28$ & $24-25$ & $22-24$ & $2-3$ \\
A. kunzeanum & $34-36$ & $22-25$ & $20-22$ & $16-22$ & $3-4$ \\
A. mucronatum & 2831 & $27-23$ & $22-24$ & $20-22$ & $2-3$ \\
A. oligophyllum & $34-39$ & $23-26$ & $20-23$ & $21-26$ & $3-4$ \\
A. papaverifolium & $40-42$ & $25-27$ & $23-25$ & $31-32$ & $2-3$ \\
A. pulchellum & $37-40$ & $27-28$ & $24-26$ & $22-27$ & $2-3$ \\
A. radicans & $32-35$ & $23-25$ & $22-24$ & $20-22$ & $1.5-2$ \\
A. sellowianum & $63-70$ & $32-35$ & $38-40$ & $30-32$ & $4-9$ \\
A. serratum & $37-40$ & $30-32$ & $26-28$ & $20-23$ & $2-3$ \\
A. trilobum & $40-43$ & 3031 & $28-30$ & $20-22$ & $4-5$ \\
A. ullbricthii & $42-55$ & $27-32$ & $28-29$ & $20-23$ & $2-6$ \\
\hline A. uniseriale & $30-31$ & $22-23$ & $23-24$ & $14-18$ & $2-3$ \\
\hline & $32-38$ & $23-25$ & $22-24$ & $18-20$ & $1.5-3$ \\
\hline
\end{tabular}

